\title{
Research on the Formability of Metallic Thin-walled Tube Hydroforming Yulai She ${ }^{1}$, Hongyi Liao ${ }^{1}$, Jianwei Liu ${ }^{1}$, YuHan LI ${ }^{1}$, Chenmei Kong ${ }^{1}$ \\ Institute of Electrical and Mechanical Engineering, Guilin University of Electronic Technology, Guilin 541004, China
}

Keywords: tube; Hydroforming; Radial Crushing bulging; Hydroforming with pulsating loading; development trend.

\begin{abstract}
Tube hydroforming is a developed and successful way for complex shapes with less operations required compared to conventional tube forming. Tube hydroforming technology is introduced briefly. Then the principle of forming, advantages and disadvantages of hydraulic bulging, Tube Hydroforming with Radial Crushing and pulse-hydraulic bulging are proposed, respectively. At last, the development trends of tube hydroforming are made a brief introduction.
\end{abstract}

\section{Introduction}

The tube hydroforming (THF) process has become more and more popular in many fields, e.g., automotive, aircraft, and aerospace industries, because of its suitability for manufacturing light and high rigidity parts. THF is advanced manufacturing technology which is produced light-weight hollow structure parts. THF is developed under this condition [1-2].

Compared with traditional crafts such as machinery, stamping, casting, forging, welding and so on, THF contains some advantages as follows [3]: (1)Lighten weight, save material. (2)Reduce the quantity of part and mold. (3) Enhance strength and rigidity of material, especially fatigue strength. (4)Decrease costs. (5)Material used more effective.

In order to obtain satisfactory formability,such as uniformity and filling property, there are more and more further researches of tube hydroforming technology. At present, the study mainly focus on hydraulic bulging, Tube Hydroforming with radial Crushing and pulse-hydraulic bulging.

\section{Tube Hydroforming technology}

The principles and Variations of hydroforming. THF is that makes the sealed tube which in the fixed mold cavity getting plastic deformations by providing high-pressure fluids, and then makes the wall completely fill the internal surface of mold cavity.

THF contains free bulging, axial pressing bulging and Extrusion forming[4]. As show in Fig 1.Free bulging means pouring high-pressure fluids into metal tube, the tube only gets the radial force, forming mainly depends on thinning of tube wall. Axial pressing bulging means on the basis of free bulging, applying axial force to the two ends of tube to accelerate metal flow into the cavity which can cover the shortage of deformation. Extrusion forming bases on axial pressing bulging, and applies radial counter-force, at last makes the wall thickness even.

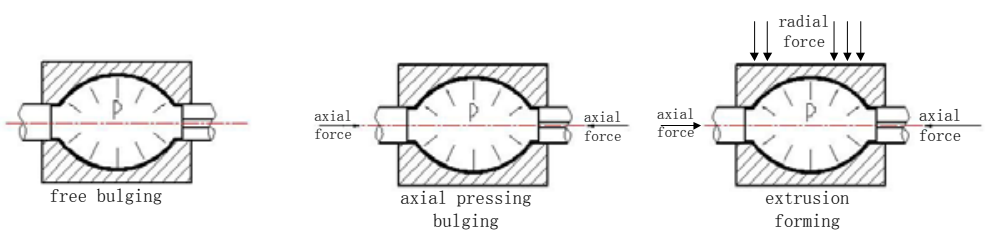

Fig1. variations of hydroforming

Main application and deficiencies.THF is widely applied to sectors such as automobile, aviation industry, civil furniture and so on. THF can lighten the structure and improve strength and rigidity of parts and manufacture circular structure which changed along the axis and hollow 
structure which has strange cross-section. Such as irregular tube in cars exhaust system, non-circle cross-section in hollow framework, sub-frame, dashboards' brackets(approximately $11 \% \sim 15 \%$ cars total mass), hollow shaft, complex tube and so on[5]. THF have a positive influence on society.

However, when part is oversize or the cross-section is irregular, due to the complex shape, the friction which the contact area between outer wall of tube and mold cavity increases. During forming, it's difficult to make material flow into mold cavity. That makes local part of the tube become thinner, distribution of thickness uneven and even burst. It needs not only lager force for forming, but also optimization for loading path is complex. Therefore, there is deeper study on new method of THF in domestic and abroad.

\section{THF with Radial Crushing}

Principle and advantage.For solving the problem that there are too much both forming pressure and clamping force, a new THF is showed up--Tube Hydraulic Forming with Radial Crushing (THFRC). As Fig2, shows the principle of THFRC .At first Let the sealed tube into the mold cavity, then inject high pressure medium (such as light engine oil, emulsion and so on) into the tube, make it free bulging under the inner pressure. When it gets a certain diameter, keep the pressure for a while. The up-die makes radial motion to provide radial force which makes the tube forming under the inner pressure and radial force. At last, we can get the forming part.

Compared to THF, THFRC has the characteristics such as lower forming liquid pressure and clamping force, uniform wall thickness distribution, fitting well and so on. Especially for the structure which has complex cross-section shape, oversize axis, and small fillet radius hollow thin-walled.

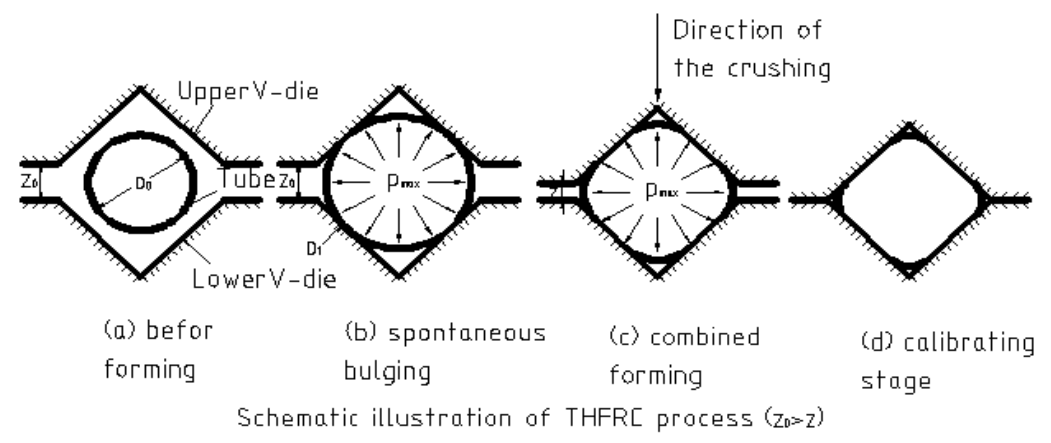

Fig2. The principle of THFRC

Research status.THFRC is an important branch of THF. In 1900s, Japanese scholar Mason[6]. pointed out that THFRC can effective make the thickness distribution better, decrease forming pressure, lower the requirements for Forming Equip. Hwang[7]et al. compared the characteristic of forming between free forming and radial pressing forming by the finite element simulation method. And the results indicated that for forming rectangular cross-section with axial pressing forming, the max forming pressure and the max clamping force just were $5 \%$ and $7 \%$ of free forming, respectively. And the thickness of part is more even, the ability for material filling and fitting is better. Yang [8] et al. simulated the process of triangle cross-section forming under radial pressure with constant pressure path by experiment and analysed max forming inner pressure, friction and constrain way of tube-end effect on THFRC of forming part's material filling, cross-section profile, thickness distribution and failure form. Lei [9] et.al studied the process of forming triangle cross-section under radial pressure with different loading path by experiment, and compared to the same condition but with free forming. Results showed that the THFRC filling better, thickness distribution more even and bulge higher. In different loading path, with the certain pressure loading path THFRC can get better filling forming and better cross-section profile, and the wall thickness more even. 


\section{Hydroforming with pulsating loading}

Principle and advantage.For principle, Hydroforming with pulsating loading is similar with free forming.The only difference is that former of the input of the hydraulic oil has a certain frequency and amplitude.

Hydroforming with pulsating loading is due to tube receives force with periodical change which makes the friction between tube wall and mold cavity decrease in the process of deformation. In the process of tube forming, small wrinkles generate when fluctuation was reduced under internal pressure, but when the internal pressure rise later, they will go away.

Under pulsating hydraulic, the occurrence and elimination of this kind of small wrinkles repeatedly perform under the pressure of hydraulic pressure changing. Using the wrinkle effectively can avoid the local part of the tube becoming thinner to make the tube becomes uniform.

Hydroforming with pulsating loading include some advantages, for example (1) Lighten parts, improve the rigidity and strength especially fatigue strength. (2) Improve products' size accuracy. (3) Reduce machining operation and model quantity. (4) Cut production cost.

Research status.Consistent research's about THF find out that loading path is one important factor of forming quality. For improving forming quality, at the beginning of 21 centuries, scholar T. Rikimaru[10] from Japan raised pulse hydraulic forming for the very first time. Amir Masound[11]et.al selected double-layer tube (out layer is brass and cooper inner) as the objection. And he simulated the process of loading which were applied pulsating hydraulic forming with max stress and liner loading with min stress by finite element and experiment. When the fillet of mold rose, the bulging became higher. Reducing the risk of wrinkle and crack can make thickness more even and better forming performance. Rong[12] et.al studied the tube pulse loading by experiment and finite element. The results showed that compared with linear loading path, pulse loading gets smaller key difference in wall thickness and the thickness is more even. With the same max hydraulic pressure and the same frequency, the amplitude more big than the height of bulging more small and the thickness more even.

For present, pulse hydraulic forming is an reasonable way for forming. But the equipment is too complex for achieving pulse hydraulic. The relationship between frequency, amplitude and material performance, size and mold process are not clear, so it needs further research.

\section{Trend of development}

(1)Due to equipment is too complex for achieving pulse hydraulic and costs are very high, we propose a new method for forming, composite hydraulic forming, forming by high impact and improve productivity.

(2) The design of mold should be reasonable and reduce typical defects. Developing design criterion of hydraulic forming mold to ensure the performance can fit design demand.

(3) The development of finite element special unit and constitutive model, develop calculated unit which can reflect performance of tube billet and establish constitutive model which can exactly reflect material characteristic on the basement of shell finite element[13].

\section{Conclusion}

THF developed so fast, and has been one important method for automobile lighter. At the moment, THF has many shortages in productive practice, there are many aspects need further research, such as how to improve productivity and develop design criterion. With the rapid development of automobile industry, the demands for hydraulic forming part will increase, and THF will develop more rapidly.

\section{Acknowledgement}


The authors truly acknowlege the funding provided by Guangxi Natural Science Foundation (Grant NO:2013GXNSFBA019245)

\section{References}

[1] Shi-jian Yuan, Yang Li, Kun Dai, Lang, Zhong-ren Wang. Internal high pressure forming of lightweight structures [J] .Material science \&Technology, 1999 (7) : 139-142.

[2] Liu, Jianwei Liu, Xinyu Yang Lianfa, Liang Huiping. Determination of flow stress of thin-walled tube based on digital speckle correlation method for hydroforming applications. International Journal of Advanced Manufacturing Technology 2013, 69(1-4), 439-450.

[3] Hongyang Li, Shi-jianYuan, Zhong-ren Wang. Hydraulic extrusion forming technology in auto industry application [J]. Journal of mould technology, 2001, 32 (4) : 7-9.

[4] Tonghai Wang ,Sheng Sun .Classification of bulging forming on tube and its deforming mechanical features [J]. Journal of forging technology, 1999, (4) : $30 \sim 32$.

[5] CHEREK H .Part cost reductions in the hydroforming processes[A] .Proceedings of 2th International Conference on Innovations in Hydroforming Technology[ C] .[ s .l .] :[ s.n.], 1997 .

[6] M Mason. Tube hydroforming using sequenced forming pressure[A]. Proceedings of the International Seminar on Recent Status and Trend of Tube Hydroforming[C]. Tokyo: Japan, 1999. 80-98

[7] Yeong-Maw Hwang,Taylan Altan.Finite element analysis of tube hydroforming process in a rectangular die[J].Finite element in Analysis and Design,2002,(39): 1071 1082.

[8] Lianfa Yang, Chen Fengjun. Design and Experimental Research on Tube Hydroforming Device in Radial Crushing [J]. Journal of north university of china (natural science edition), 2010, 31 (6) : 562-567.

[9] Pan Lei. Investigation on the Formability of a Tube in Hydroforming with Radial Crushing Under the Simple Loading Paths [D] : guilin university of electronic technology, 2012.30-50.

[10]T. Rikimaru, M. Ito. Hammering hydro-forming of tubes[J]. Press Working. 2001, 39 (7): 58-65.

[11] Mohsen Loh-Mousavi, Amir Masoud Mirhosseini and Ghasem Amirian. Investigation of modified bi-layered tube hydroforming by pulsating pressure[J]. Engineering materials.2011 486:5-8.

[12] Haisong Rong . Investigation on Deformation Behavior of Tube Hydro-forming with Radial Crushing under Pulsating Pressure[D] Guilin:guilin university of electronic technology, 2014,23:429-438.

[13] Hongyang Li ,Haijun Liu,Haiyuan Lv ,Wang Xie, Yichen Lu.Present State and Perspective of Tube Hydroforming in China[J]. The Chinese mechanical engineering. 2006:55 to 59. 